\title{
Acute neurotoxicity following fenpyroximate poisoning
}

\author{
A N KuruppuArachchi ${ }^{1}, \mathrm{~K}$ T Sundaresan ${ }^{1}$, M Umakanth ${ }^{1}$, T Thivakaran ${ }^{2}$
}

Ceylon Medical Journal 2018; 63: 186-187

DOI: http://doi.org/10.4038/cmj.v63i4.8770

\section{Introduction}

Fenpyroximate is a potent inhibitor of mitochondrial proton-translocating NADH-quinoneoxidoruductase (complex 1) [1]. It is used in the agricultural sector as an acaricide. Acute and chronic toxicity studies of fenpyroximate in laboratory animals have been conducted by oral lavage. There were no treatment-related deaths and neuropathological evaluation in rats did not reveal any treatment-related lesions [2]. Laboratory testing has documented limited toxicities particularly irritation to the skin and eyes. LD50 for this product is $245 \mathrm{mg} / \mathrm{kg}$ for mammalians [3]. Laboratory studies have classified fenpyroximate (along with rotenone) as a Complex I inhibiting pesticide that is neurotoxic to astrocytes when cultured by in vitro bioassays [4]. Despite its worldwide usage as an insecticide, there are only few reported cases of its human toxicity. Lee et al [5] reported a case of near fatal fenpyroximate poisoning that resulted in severe lactic acidosis and cardiac arrest needing percutaneous cardiopulmonary support and therapeutic hypothermia. The authors were unable to find any case of acute neurotoxicity as a presentation following fenpyroximate poisoning in either Poisoning Center data base in Sri Lanka or in a search of the published literature using the PUBMED data base.

\section{Case report}

A 31-year-old single sales assistant in a grocery business was transferred from a peripheral hospital following self-ingestion of around $100 \mathrm{ml}$ of $50 \mathrm{~g} / \mathrm{l}(5 \mathrm{~g})$ fenpyroximate (Mitigate) (Figure 1), swallowed four hours earlier. Gastric lavage and activated charcoal had been administered prior to transfer. On admission to the emergency department he complained of progressive bilateral lower limb weakness and unsteady gate. He was well oriented and afebrile and denied any previous history of neurological disorders.

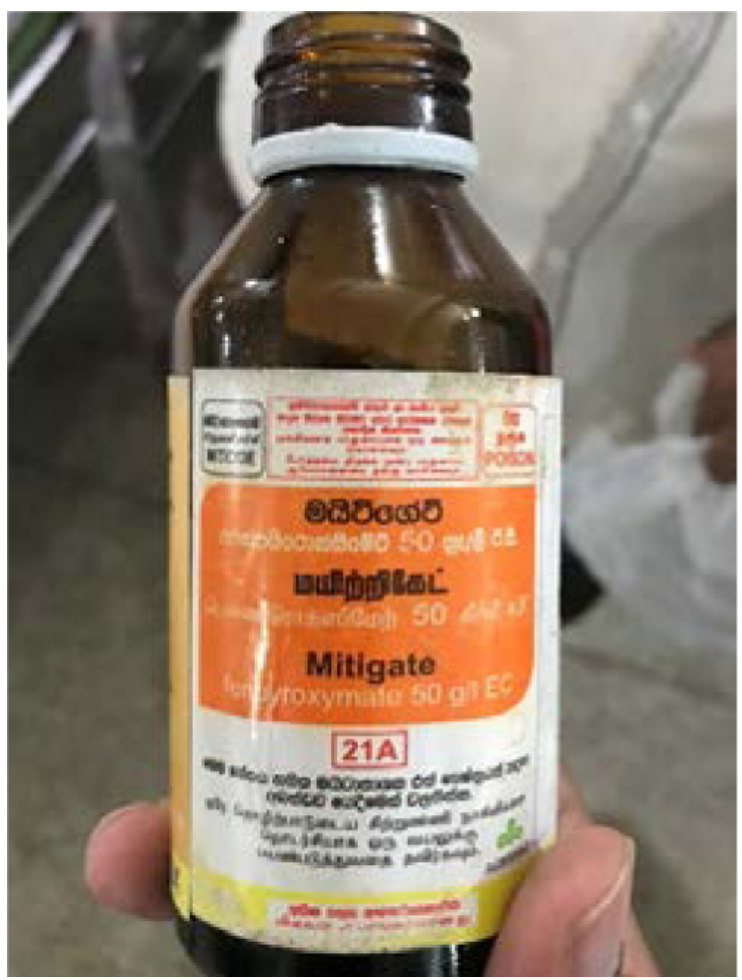

Figure 1. An illustration of the container of the pesticide with minimal labeling to indicate its poisonous nature.

On examination, MRC grade 4 weakness was noted in both lower limbs. There was sensory loss of all modalities and a sensory level was demonstrated at T4 level. His upper limb and cranial nerve examination were normal. His blood pressure was $130 / 80 \mathrm{mmHg}$ and was stable throughout. Pulse rate was 88 beats per minute. Oxygen saturation was $100 \%$ on room air. His respiratory and abdominal examination was unremarkable.

Three hours after the admission he developed transient self-limited bilateral partial ptosis and absent gag

${ }^{1}$ Department of Internal Medicine, Teaching Hospital Batticaloa, ${ }^{2}$ Department of Neurology, Teaching Hospital Batticaloa.

Correspondence: ANK e-mail: <nadee86sha@gmail.comm>. Received 13 August 2018 and revised version accepted 03 October 2018.

This is an open-access article distributed under the terms of the Creative Commons Attribution License, which permits unrestricted use, distribution, and reproduction in any medium, provided the original author and source are credited. 
reflex which resolved completely over next 24 hours. There was no bulbar weakness. At this point the patient was transferred to the ICU for monitoring.

His full blood count revealed mild neutrophil leukocytosis, ESR was $12 \mathrm{~mm} / 1^{\mathrm{st}} \mathrm{hr}$ and CRP was $22 \mathrm{mg} / \mathrm{dl}$. Serum sodium was $138 \mathrm{mmol} / \mathrm{L}$ and serum potassium was $4.4 \mathrm{mmol} / \mathrm{L}$. Arterial blood gas showed mild hyperventilation, no acidosis, and lactate level was $1.7 \mathrm{mmol} / \mathrm{L}$. His liver and renal functions were within the normal range.

On the next day his ptosis and gag reflex were completely resolved. Lower limb weakness also began to improve. Pain and temperature sensation was regained and joint position sensation was the last to improve which took almost three days for complete resolution. Electromyography and nerve conduction studies were completely normal. He was discharged without any residual deficit.

As a village grocer serving an agricultural society our patient had access to mitigate that was freely available in his grocery shop. It was not clear whether there was an intention to commit suicide. However, appropriate counseling was offered to avoid future episodes. One month later he was reassessed in the ward and detailed neurological testing did not reveal any residual abnormality.

\section{Discussion}

Pesticide ingestion is the commonest method of nonfatal self-poisoning in Sri Lanka, being more prevalent among young males [6]. In a study of choice of poison for intentional self-poisoning in rural Sri Lanka, Eddleston et al [7] did not refer to fenpyroximate as an agent used for self-poisoning.

Acute neurotoxicity following fenpyroximate poisoning is a novel experience for clinicians based at rural hospitals serving farming communities. Our index case manifested as an acute cord syndrome with possible transient brainstem dysfunction. Neurological manifestations were patchy and self-limited. Fortunately, more grave manifestations like lactic acidosis and cardiac toxicity did not develop in this patient, and his biochemistry profile remained normal. In the absence of an antidote to fenpyroximate the case was managed conservatively. We were unable to organise an MRI investigation in our unit in Batticaloa though this was indicated for a case of this nature with brain stem and spinal cord involvement. This as a limitation of our case study.

According to the Threshold of Toxicological Concern (TTC) fenpyroximate is classified as a Class III substance by the Cramer scheme suggesting significant toxicity or to have reactive functional groups (3). Complex I-inhibiting neurotoxins, such as the pesticide rotenone is known to cause neuronal death and parkinsonism in animal models [4]. Furthermore, pesticide exposure is epidemiologically-linked with an increased risk for Parkinson's disease [8]. Parkinson's disease brains show evidence of mitochondrial respiratory complex I deficiency, oxidative stress, and neuronal death. Thus it is likely that fenpyroximate being a potent inhibitor of mitochondrial activity may contribute to neurotoxicity.

The case described allows us to draw attention to an uncommon clinical presentation due to pesticide poisoning in an adult Sri Lankan male. Strict labeling and improved public awareness on dangers of agrochemicals to humans should be undertaken to reduce the incidence of selfpoisoning, in developing agricultural countries such as Sri Lanka. Usefulness of developing an antidote to mitigate to be administered to patients brought to EDs is highlighted by this case.

\section{Conclusion}

Fenpyroximate poisoning can give rise to acute neurotoxicity in humans. To ascertain the exact clinical manifestations of the condition, and the likelihood of permanent neurological damage from this agent we need further evaluation of similar cases.

\section{Disclosure statement}

We have no conflict of interest to declare. This research received no specific grant from any source.

\section{References}

1. Pesticideinfo.org. (2018). Fenpyroximate - toxicity, ecological toxicity and regulatory information: Available at http://www.pesticideinfo.org/Detail_ Chemical.jsp? Rec_Id=PC36332 [Accessed 2 Apr. 2018].

2. Barnett JF. Acute neurotoxicity study of fenpyroximate in $\mathrm{Crl}$ : $\mathrm{CD}(\mathrm{SD})$ rats by oral gavage; Charles River Laboratories, Preclinical Services, Pennsylvania, Horsham, PA, Monroeville, PA; Study No. 20004074; 3/29/11.

3. Pesticide Properties Data Base (PPDB); URL: https:// sitem.herts.ac.uk/aeru/ppdb/en/

4. Mullett, S. and Hinkle, D. DJ-1 deficiency in astrocytes selectively enhances mitochondrial Complex I inhibitorinduced neurotoxicity. Journal of Neurochemistry 2011; 117(3): 375-87.

5. Lee H, Lee B, Jeung K, Lee G, Jung Y, Jeong I. A case of near-fatal fenpyroximate intoxication: The role of percutaneous cardiopulmonary support and therapeutic hypothermia. Clinical Toxicology 2012; 50(9): 858-61.

6. Rajapakse T, Griffiths KM, Christensen H. Characteristics of non-fatal self-poisoning in Sri Lanka: a systematic review. BMC Public Health 2013; 13: 331.

7. Eddleston M, Karunaratne A, Weerakoon M, Kumarasinghe S, Rajapakshe M, Sheriff MH, Buckley NA, Gunnell D. Choice of poison for intentional self-poisoning in rural Sri Lanka. ClinToxicol (Phila) 2006; 44(3): 283-6.

8. Sherer T, Betarbet R, Testa C, Seo B. Mechanism of toxicity in rotenone models of Parkinson's disease. J Neuroscience 2003; 23(34): 10756-64. 